\title{
An Overview of History, Evolution, and Manufacturing of Various Generations of Vaccines
}

\author{
Alireza Tahamtan, ${ }^{1,2}$ Javad Charostad, ${ }^{1}$ Seyyed Javad Hoseini Shokouh, ${ }^{3}$ and Mohammad Barati ${ }^{3,}$ \\ ${ }^{1}$ Department of Virology, School of Public Health, Tehran University of Medical Sciences, Tehran, Iran \\ ${ }^{2}$ Department of Microbiology, Golestan University of Medical Sciences, Gorgan, Iran \\ ${ }^{3}$ Infectious Diseases Research Center, AJA University of Medical Sciences, Tehran, Iran \\ "Corresponding author: Mohammad Barati, Infectious Diseases Research Center, AJA University of Medical Sciences, Tehran, Iran. Tel: +98-2143822990, E-mail: \\ mbaratim@gmail.com
}

Received 2017 April 30; Revised 2017 September 03; Accepted 2017 September 05.

\begin{abstract}
Vaccination has had great success in history of medicine with both infectious and non-infectious diseases listed in the realm of vaccines. The significant decrease in deaths from infectious and non-infectious diseases by development of vaccines has had a huge impact on the world health. Since the 20th century, for preventive and treatment purposes, there has been an emergence of new and diverse range of vaccines in medicine. Also, there are ongoing extensive efforts to increase the efficiency and performance of vaccines and reduce the risks, which are associated with humans. Despite of the development of advanced and effective vaccines in the recent decades, vaccines should be presented with different mechanisms to prevent epidemic and pandemic diseases. Vaccination has received the greatest attention in military aspects and several vaccines have been developed. Therefore, it seems that the study of different types and generations of vaccines, understanding their evolution, and production and development of new and modern vaccine is essential.
\end{abstract}

Keywords: Vaccines, Medical Science, Prevention, Treatment

\section{Introduction}

Infectious diseases that are caused by pathogens are one of the most important challenges faced by humans. In the past, diseases due to severe complications and high mortality have been horrific for people (1). Using vaccines in order to prevent and control diseases has been one of the greatest achievements in the history of medicine (2). After the presentation of vaccines against infectious diseases, the world health organization (WHO) reported on the notable decline in mortality and the complications of diseases, which are caused by vaccine's stability (3). Records signify the use of vaccines in medical interventions, preventing the death of 5persons per minute and 25 million deaths during the period between 2011 and 2020 (4). There are many advantages in the use of vaccines in comparison to common chemical drugs, such as longtime immunity, preventive and curative performance, and lack of drug resistance with the use of vaccines (5).

Today, in addition to infectious diseases, vaccines can prevent and treat non-infectious diseases like cancer, and researchers are trying to produce vaccines for different types of cancers. Furthermore, diseases associated with military subjects are in the aspect of vaccination. For example, in the United States, adenoviruses 4 and 7 vaccine has been used for military subjects. In general, an ideal vaccine should have the following properties, i) maximum safety, ii) a small amount of imunogene that could lead to longterm protection against pathogens, iii)affordability and effectiveness, iv) be operative and useful in prevention and treatment, v) be used in a single dose, vi) easy prescription, vii) be polyvalent and allow protection against various aspects of the pathogen (6).

Since the development of vaccines, they have been considered as a proper strategy to reduce health and treatment costs, and researchers are trying to increase the number of diseases that could be prevented by vaccination (7). This study aimed to review the history and development of vaccines, types of vaccines, specifications, and their usage.

\subsection{History of Vaccine Development}

The immunization history of individuals was initiated with observations and experiences of people of China and Middle East, who lived between the 12th century and 15th century $(1,8)$. During this time, they used the skin or pustule liquid of patient, who were diagnosed with Smallpox for immunization purposes. In the inoculation process, they used the smallpox virus to protect people against their next exposure to the virus. Although this process could lead to protection against disease, there is a high risk of developing the diseases and therefore death (9).

After China and Middle East, this treatment method developed in other areas, such as Europe in the 17th century. 
The first scientific study about this method was done by Edward Jenner, while he used the Cowpox instead of human Poxvirus for injection in humans, and this was the beginning of vaccine development. In spite of Jenner's historic achievement, due to a lack of adequate information and basic knowledge on microbes and microbiology, no new vaccines were introduced until the next century. By the end of the 19th century and the rise of scientists, such as Louis Pasteur, Robert Koch, and Paul Ehrlich and through the discovery and development of immunization science, began a new era in vaccine studies (10). This era led to the production of new vaccines against pathogens and extensive researches conducted around the world. During these researches, vaccines were produced and marketed until 1930 against various factors, such as Rabies, Typhoid, Diphtheria, Tuberculosis, Tetanus, and Pertussis $(11,12)$. In 1931, according to the valuable findings of Louis Pasteur on growing the virus in chicken embryos, a new era of mass production of vaccines began by applying multiple techniques. During years 1930 to 1950, especially during World War II, military targets created high motivation in the development of vaccines. Also, from that period onwards, the support of foundations and public agencies, such as WHO and research institutes increased significantly. Vaccines, such as Polio, Japanese encephalitis B, and Influenza were produced at this time $(13,14)$. After World War II, targeting vaccination programs became to a universal tool for improving public health (2). In the wake of the global vaccination program and the supply of vaccines around the world, related mortality was significantly reduced, and Smallpox and Polio were eradicated $(15,16)$. In the second half of the 20th century, scientific findings, which are associated with screening and industrial production of vaccines, led to the development of a new generation of vaccines and this period became known as the golden age of vaccine science. This period began by producing vaccines against Measles, Rubella, and Mumps in the 1960s, continued with the production of Chickenpox vaccine, and inactivated Japanese encephalitis in the 1970s (17). Cultivation techniques under controlled conditions have been used in the process of mass production of vaccines. After this period, in the 1980s, the conjugated vaccine of capsular polysaccharide and proteins were developed (18). By using conjugated vaccines with carrier proteins, the problem of dysfunctional immune response against carbohydrates antigens was solved. Progress in the field of genetic engineering led to the development of recombinant vaccines. These vaccines enhanced the effectiveness of the immune response and safe use of antigens for immunogenicity in a group of pathogens like hepatitis B $(19,20)$. Eventually in the 1990, vaccines evolution entered a bold and surprising final phase with the development of vaccines, called
DNA vaccines. At this time, Wolf reported that intramuscular injection of DNA leads to an immune response, and researchers found that intramuscular injection of plasmid carrying the Influenza virus gene leads to stimulates cytotoxic lymphocyte response against the influenza antigen (21). This vaccine is a promising approach to deal with infectious and non-infectious diseases. The main point in utilizing these vaccines is their usage against non-infectious diseases, such as tumors. Since the presentation of these vaccines, extensive advancements have been done in hope of developing vaccines against diseases that human have not been able to prevent or treat, such as human immunodeficiency virus (HIV) and Malaria (22).

\subsection{The Process of Vaccine Production}

As drug production, the production and evaluation of vaccines includes 3 steps: identify and producing antigens or imunogene, tests on animal models, and clinical trials. In the first phase of the clinical trial, safety and tolerability of the vaccine is evaluated at both limited and extensive levels, and the range of doses or repeated doses of the vaccine is also measured $(23,24)$. These trials are usually designed as randomized and cohort studies and use placebo. In the second phase, the vaccine safety and immunogenicity of active ingredient should be evaluated in the target population and the proper dose of vaccine should be identified. In the third and final phase, necessary measures should be taken for obtaining the license, and the efficiency and safety of the vaccine formulation should be evaluated on the large scale in the target population. Most of the clinical trial steps are the same during the 2nd and 3rd phase, yet the basic difference is related to the size of the evaluated population. However, there is one more step to obtain necessary authorizations for commercialization of the vaccine, during which the vaccine is tested in a region, city or country (24). On average, all vaccine development processes take 16 years and cost about a billion dollars in the US (Figure 1). Before clinical evaluation of the vaccine and supply to the market, there should be a certain program to make sure the efficiency and success of the vaccine. This program should include cases as the target population (healthy subjects with characteristics statistics) considering socio-cultural factors, and target disease risk and vaccine safety, the incidence of target disease and environmental factors, dosage, injection procedure and a safety induction program for the group should be evaluated (23).

\subsection{Type of Vaccines and Their Classification}

In general, vaccines are divided to 3 generations based on the process of evolution and there are specific characteristics and covering spectrum in each generation, as explained in Table 1. 


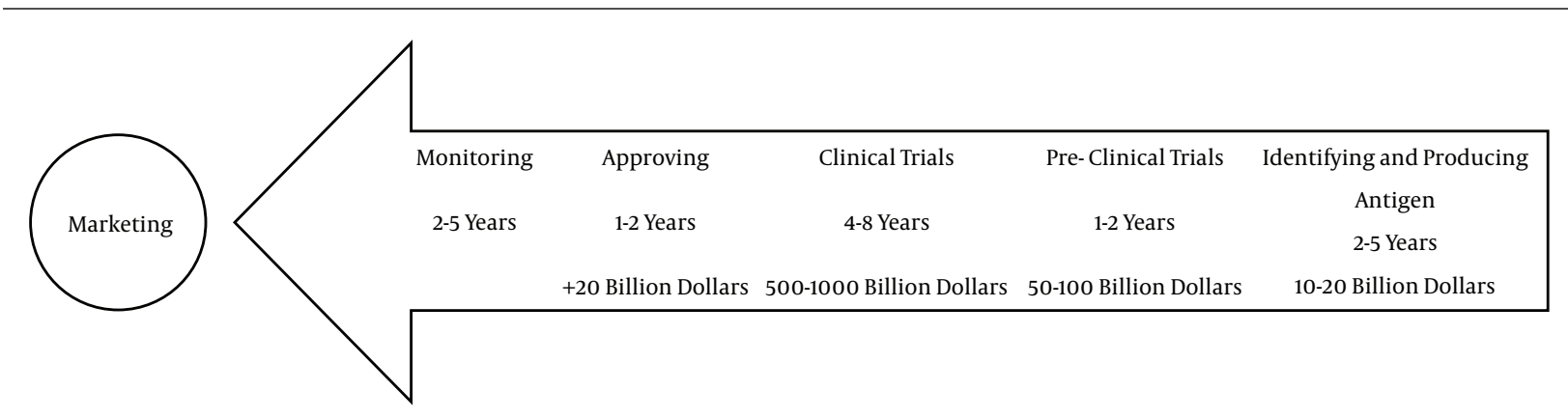

Figure 1. The Process of Vaccine Production

Table 1. Different Types and Generations of Vaccines

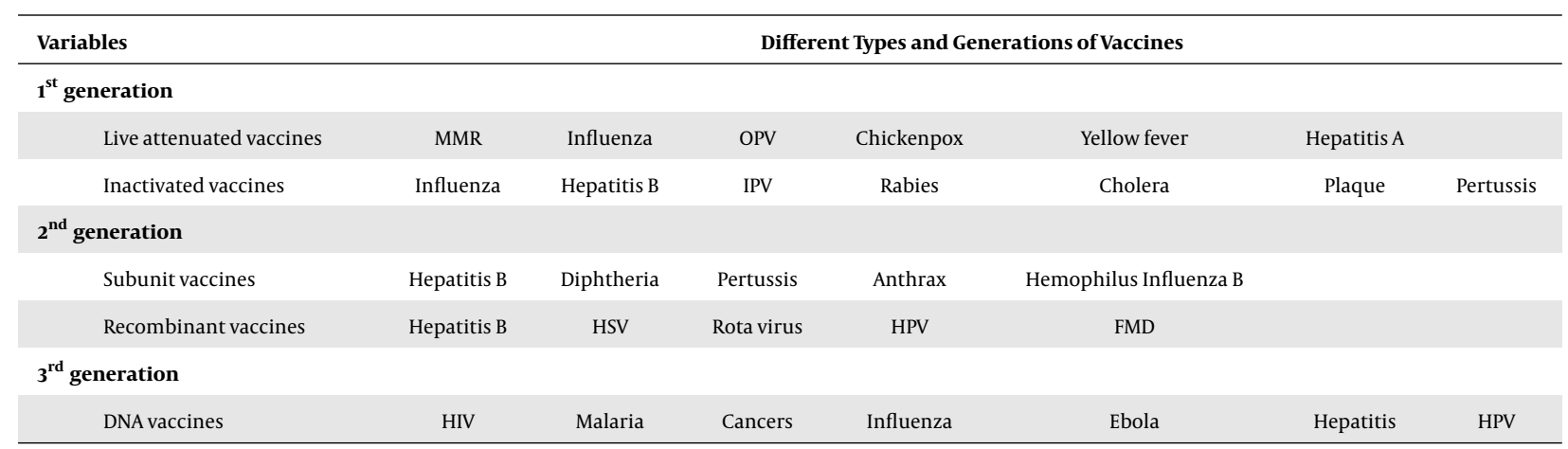

\subsubsection{First-Generation Vaccines}

Attenuated and inactivated vaccines are identified in the first generation, which use a primary method in their production. Attenuated pathogens, full organisms or inactivated bacterial toxin, which are effectively immunogenic, are used in making these vaccines. There are some advantages in these kinds of vaccines due to their high ability to stimulate innate immunity, induction of long-term protection, easy production, and low production costs (25). However, there are some disadvantages in this generation, such as inducing disease due to the use of complete pathogen (live or inactivated) and virulence recursively of the pathogen in the host body $(26,27)$. This type of vaccine is known as a traditional vaccine.

\subsubsection{Attenuated Vaccines}

These kinds of vaccines, which are known as live attenuated vaccines, contain the major types of pathogens, which have all the pathogenic features of the main organisms and have been attenuated on laboratory conditions. These vaccines are useful as they have the ability to emulate the infection process, which could lead to induction of potent antibody responses and cellular immunity. The advantages of attenuated vaccines include long-term safety and no need for a booster dose (an additional dose)(3). The procedures of attenuating an organism by pathogenic characteristic could be different, for example in Jenner's approach on Pox, he used a kind of pathogenic virus in a different host, which did not have any pathogenic characteristic for humans (9). On the other hand, weak pathogenic strains like type 2-poliovirus vaccine could also be used. In addition, the attenuation of pathogens could be obtainable by imposing strict conditions on pathogens (cold adaptation of influenza virus) (28).

The triple vaccine of Measles, Mumps and Rubella (MMR) is a kind of prominent attenuated vaccine, which was supplied in the United States in 1971 (29). This vaccine contains all 3 types of viruses, which have been attenuated. Each of these strains is obtained (Measles (Schwarz), Mumps (RIT4385) and Rubella (Wistar RA27/3)) separately from in vitro embryo or MRC5 human diploid cells (30). The Polio (OPV), Influenza, Yellow fever, and Hepatitis A are the other known vaccines in this group.

\subsubsection{Inactivated Vaccines}

In comparison with attenuated vaccines, the main advantage of inactivated vaccines is their usage of inactivated or killed pathogens, which cause an increase in 
safety and immunity level. However, there are some concerns, such as return of the virulence characteristics of the pathogen, which lead to host disease and because of non-proliferation, pathogens clear rapidly from the body, which could decrease the efficiency and effectiveness of vaccines in comparison with attenuated vaccines. Hepatitis $A$ is an inactivated vaccine that was produced by using hepatitis A virus (type RG-SB), which is inactivated by formalin (31). The use of inactivated vaccines started in early 1940s, when Brazilian researchers have pioneered in this field (5). Cutaneous Leishmaniasis vaccine is an inactivated vaccine, on which extensive studies have been done in Iran. Leishmaniasis inactivated vaccine was first evaluated by Mohebali et al. in Iran (32). In a clinical trial conducted in Bam, Kerman province, in 1998, Leishmaniasis attenuated vaccine (ALM + BCG) was used for individual immunization against tropic Leishmaniasis (33).

\subsubsection{Second-Generation Vaccines}

The first generation was made by live attenuated pathogens, which had the possibility of returning to natural and infectious forms and finally disease. Thus, to solve this problem, researchers and scientists offered secondgeneration vaccines (34). The basis of this generation was subunit elements, recombinant or synthetic proteins, nonprotein antigens, and expressed bacterial imunogene or viruses, which include numerous molecules and epitopes of different species and strains of pathogens (6). The subunit, conjugated, and recombinant vaccines are in this generation.

\subsubsection{Subunit Vaccines}

Instead of the complete pathogen, parts are used for the production of these kinds of vaccines. Subunit vaccines have one or more protein-peptide or polysaccharide, which are naturally found in the pathogenic structure (35). Due to partial use of pathogen in subunit vaccines, they do not have the ability to proliferate and thereby make unintended response. Because of their immunity and low production costs, they are viable alternatives to the traditional vaccines (27). Bacterial subunit vaccines are divided to 2 basic types; the first type is Toxoid, in which toxins are the main pathogenic factor. Toxins are inactivated by using inactive formaldehyde to change them to non-toxin forms (toxoid) and then they could be used for vaccination purposes. The close resemblance of toxoid and toxin enables the immune system to neutralize original toxins by antibodies. Tetanus, diphtheria, and pertussis toxoid vaccines could be considered in this category. The second type is made of polysaccharide capsule of encapsulated bacteria. Because of the combination of antigen (usually bacte- rial polysaccharide) and carrier protein, they are known as conjugate $(36,37)$.

\subsubsection{Conjugated Vaccines}

Polysaccharide antigens are large molecules with repetitive epitopes, and antigen presenting cells are not enable to process them, thus the antibody response occurs without the participation of $\mathrm{T}$ cells against them and causes a rise of antibody response at a small dose and short-time period (38-40). These responses were not able to make immune memory and affinity maturation for these kinds of infections (41). Unlike polysaccharide antigens, proteins could be processed well by antigen presenting cells, and make long-term response and immunological memory. In 1929, for the first time, Avery and Goebel used protein to increase the immunogenicity of polysaccharide antigens. They observed that weak immunogenicity of polysaccharide antigen of streptococcus pneumonia type 3 increased by its connection to the carrier protein in rabbit. These observations led to a foundation for producing modern conjugate vaccines (42). In 1987, hemophilus influenza B (HIB) was the first conjugate vaccine, which could obtain license for medical usage and was later used for immunization of infants. The HIB conjugate formulation made by different types of carrier proteins contains tetanus toxoid, diphtheria toxoid, diphtheria toxin mutant, and outer membrane protein, which caused increases in the quantity and quality of its immunogenicity (43). The success of conjugate vaccine in reducing the incidence of disease in children led to an acceleration of their development for preventing encapsulated pathogenic bacteria (44). The streptococcus pneumonia, meningococcal, and HIB belong to this group.

\subsubsection{Recombinant Vaccines}

Today, the rise of genetic engineering and molecular biology has had great impact on development and manufacturing process of vaccines. Specific antigenic microbes have high power to arouse the immune response against pathogens. Currently, the sequence of the pathogenic protein antigens could be obtainable by sequencing genes of the main antigen, and producing them synthetically via recombinant DNA technology. Hepatitis B is the first and one of the most successful examples of synthetic vaccines. The surface antigen of this virus (HBsAg) is very immunogenic and effective, and able to produce high levels of antibody in the body (45). In the past, for providing hepatitis B vaccine, HBsAg was purified from the plasma of infection carriers and used for vaccination; of course there were some extensive restrictions in purification, such as difficult conditions and contaminated plasma. In order to make recombinant hepatitis B vaccine, recombinant HBsAg is ex- 
pressed in cells that have a powerful expression system (such as yeast) leading to the production of virus-like particles by HBsAgs, which are highly immunogenic. Since these particles have no genome, they do not create disease and lead to effective and powerful response against the main pathogen $(46,47)$. Other kinds of common vaccines are anti-herpes simplex virus, anti-rotavirus, and anti-HPV vaccines.

\subsubsection{Third-Generation Vaccines}

Immunogenic potential administration of a plasmid containing a gene encoding the antigen, known as genetic vaccines, is categorized as third generation vaccines, and is a valuable method, which has been considered by researchers since the beginning of 1990s. Different names have been given for this kind of vaccine, such as DNA vaccines, RNA vaccines, and plasmid vaccines. The expertise committee of WHO vaccination in 1996 chose nucleotide acid that includes both DNA and RNA vaccines. Furthermore, genetic immunization and DNA immunization terms were used for this type of immunization.

DNA vaccines include direct injection of plasmid containing the encoding gene of the considered antigen, which is expressed in the cells with the aid of specific promoter that causes induction of the immune system. Therefore, instead of prescribing recombinant protein needed to stimulate the immune system (such as hepatitis B), it will be produced in the body. The expressed protein, that are in the natural form, stimulate cellular and humeral immunity during different stages. The dendritic cells play an essential role in providing antigen to immune cells (48, 49). The advantage of DNA vaccines are: 1 ) they do not have the limitation of common recombinant protein and peptide vaccines, 2) are able to immunize against several different strains of qualified antigen diversity, 3) produce antigen in a natural form and properly deliberated to the immune system, 4) are able to stimulate the humeral, cellular, and mucosal immunity, 5) can make sustainable immunity, 6) cause a lack of immune response to the injected vector and not generate antigen against transfected cells, 7) there is no possibility to activate attenuated vaccines and infection risk, 8) it has been possible to produce multiple vaccines, 9) have ease, speed of mass production and similar stages of vaccine production, 10) the quality control is an easy process, and 11) it is stable in various temperatures and the cold chain is not required for maintenance (49).

In addition to extensive benefits, DNA vaccines have some serious limitations, such as 1) the ability to be only used for protein antigens, 2) effect cells growth by controlling genes, 3) are capable of joining the host genome and inducing tumors, and 4) can cause autoimmune diseases (50).
In the recent years, extensive research projects have been done on the use of this generation of vaccines for hepatitis, HIV, influenza, and Ebola (51). Nevertheless, they have not received indispensable license for human usage. Several clinical trials are ongoing on the use of these vaccines in cancers and human immunodeficiency virus (HIV). Some of the DNA vaccines obtain indispensable license in veterinary usage (52). In Iran, several studies have been done on the usage of anti-tumor vaccines, such as that of Tahamtan et al., in 2014, on the usage of DNA vaccines against cervical cancer induced by papilloma virus type 16 in a mouse model. In this research, a tumor-associated antigen was used for cervical cancer immunotherapy in the form of HPV DNA vaccine (53). In this study, the nanoparticles of chitosan was used as a carrier for DNA vaccines and for enhancement of anti-tumor effects, and the results indicate the ability of this compound on HPV cancer treatment (54).

In the 21st century, expanding new vaccine target population in age groups and specific human population was one of the most important aspects of vaccination (52). In order to achieve such a goal, increase of clinical trials on populations around the world and advanced technical facilities for the successful production of new vaccines were necessary $(55,56)$. Today, there are some instructions for vaccine usage on different age groups and for people with special conditions.

\section{Conclusions}

In the 21st century, the world became informed about the importance of preventing serious infectious diseases, such as HIV, tuberculosis, malaria, and non-infectious diseases like different types of cancers, which make financial and casualty issues for the public health system. Clearly, preventing diseases is much more effective and less costly than treatment after obtaining the disease; therefore, vaccine is the most important and powerful instrument for humans in order to prevent diseases and the development of vaccination is very important. Undoubtedly, the quality and quantity of clinical vaccines will be widely promoted in the future. The aim of new strategies is increased safety, efficiency, and stability of vaccines in the future. By expanding our knowledge in microbiology, immunology, molecular biology, genetic engineering, and manufacturing techniques for vaccine, the path of advanced generation of vaccines, which are able to prevent and treat such diseases, becomes easier than before. 


\section{References}

1. Hilleman MR. Vaccines in historic evolution and perspective: a narrative of vaccine discoveries. Vaccine. 2000;18(15):1436-47. doi: 10.1016/S0264-410X(99)00434-X. [PubMed: 10618541].

2. Bloom DE, Canningm D. , Weston M. The value of vaccination. Henley on Thames: World Economics; 2005.

3. Clem AS. Fundamentals of vaccine immunology. J Glob Infect Dis. 2011;3(1):73-8. doi: 10.4103/0974-777X.77299. [PubMed: 21572612].

4. Tahamtan A, Charostad J, Shokouh H, Javad S, Barati M. An overview of different types of immune stimulating adjuvants and their application. Paramedical Sci Mil Health. 2016;11(3):43-51.

5. Nagill R, Kaur S. Vaccine candidates for leishmaniasis: a review. Int Immunopharmacol. 2011;11(10):1464-88. doi: 10.1016/j.intimp.2011.05.008. [PubMed: 21616175].

6. Coler RN, Reed SG. Second-generation vaccines against leishmaniasis. Trends Parasitol. 2005;21(5):244-9. doi: 10.1016/j.pt.2005.03.006. [PubMed: 15837614]

7. Ehreth J. The global value of vaccination. Vaccine. 2003;21(7-8):596600. doi: 10.1016/S0264-410X(02)00623-0. [PubMed:12531324]

8. Leung AKC. History of Vaccine Development. Springer; 2011. pp. 512."Variolation" and Vaccination in Late Imperial China, Ca 1570-1911.

9. Riedel S. Edward Jenner and the history of smallpox and vaccination. Proc (Bayl Univ Med Cent). 2005;18(1):21-5. [PubMed: 16200144].

10. Plotkin SA. History of vaccine development. Springer Science \& Business Media; 2011.

11. Wever PC, van Bergen L. Prevention of tetanus during the First World War. Med Humanit. 2012;38(2):78-82. doi:10.1136/medhum-2011-010157. [PubMed: 22543225].

12. Rappuoli R, Malito E. Corynebacterium diphtheriae and Related Toxigenic Specie. Springer; 2014. pp. 225-38.History of Diphtheria Vaccine Development.

13. Lyons A, Longfield J, Kuschner R, Straight T, Binn L, Seriwatana J, et al. A double-blind, placebo-controlled study of the safety and immunogenicity of live, oral type 4 and type 7 adenovirus vaccines in adults. Vaccine. 2008;26(23):2890-8. doi: 10.1016/j.vaccine.2008.03.037. [PubMed: 18448211]

14. Hoyt K. Vaccine innovation: lessons from World War II. J Public Health Policy. 2006;27(1):38-57. doi: 10.1057/palgrave.jphp.3200064. [PubMed: 16681187] discussion 58-60.

15. Ehreth J. The value of vaccination: a global perspective. Vaccine. 2003;21(27-30):4105-17. doi: 10.1016/S0264-410X(03)00377-3. [PubMed: 14505886].

16. Rappuoli R. Bridging the knowledge gaps in vaccine design. Nat Biotechnol. 2007;25(12):1361-6. doi: 10.1038/nbt1207-1361. [PubMed: 18066025].

17. Hilleman MR. History of Vaccine Development. Springer; 2011. pp. 207-18.The Development of Live Attenuated Mumps Virus Vaccine in Historic Perspective and Its Role in the Evolution of Combined Measles-Mumps-Rubella.

18. Avery OT, Goebel WF. Chemo-Immunological Studies on Conjugated Carbohydrate-Proteins : V. The Immunological Specifity of an Antigen Prepared by Combining the Capsular Polysaccharide of Type Iii Pneumococcus with Foreign Protein.JExp Med. 1931;54(3):437-47. doi: 10.1084/jem.54.3.437. [PubMed: 19869930].

19. Adkins JC, Wagstaff AJ. Recombinant hepatitis B vaccine: a review of its immunogenicity and protective efficacy against hepatitis B. BioDrugs. 1998,10(2):137-58. doi: 10.2165/00063030-199810020-00005. [PubMed: 18020591].

20. Zhou J, Sun XY, Stenzel DJ, Frazer IH. Expression of vaccinia recombinant HPV 16 L1 and L2 ORF proteins in epithelial cells is sufficient for assembly of HPV virion-like particles. Virology. 1991;185(1):251-7. doi: 10.1016/0042-6822(91)90772-4. [PubMed: 1656586].

21. Kutzler MA, Weiner DB. DNA vaccines: ready for prime time? Nat Rev Genet. 2008;9(10):776-88. doi: 10.1038/nrg2432. [PubMed:18781156].
22. Teimourpour R, Meshkat Z, Arzanlou M, Peeridogaheh H. DNA vaccine: The third generation vaccine [In Persian]. Qom Univ Med Sci J. 2016;10(10):86-99.

23. Goetz KB, Pfleiderer M, Schneider CK. First-in-human clinical trials with vaccines-what regulators want. Nat Biotechnol. 2010;28(9):9106. doi: 10.1038/nbt0910-910. [PubMed: 20829825].

24. Han S. Clinical vaccine development. Clin Exp Vaccine Res 2015;4(1):46-53. doi: 10.7774/cevr.2015.4.1.46. [PubMed: 25648742].

25. Noazin S, Modabber F, Khamesipour A, Smith PG, Moulton LH Nasseri K, et al. First generation leishmaniasis vaccines: a review of field efficacy trials. Vaccine. 2008;26(52):6759-67. doi: 10.1016/j.vaccine.2008.09.085. [PubMed: 18950671].

26. Huang DB, Wu JJ, Tyring SK. A review of licensed viral vaccines, some of their safety concerns, and the advances in the development of investigational viral vaccines. J Infect. 2004;49(3):179-209. doi: 10.1016/j.jinf.2004.05.018. [PubMed:15337336].

27. Robinson HL, Amara RR. T cell vaccines for microbial infections. Nat Med. 2005;11(4 Suppl):S25-32. doi: 10.1038/nm1212. [PubMed 15812486].

28. Organization WH . Releve` e'pide`miologique hebdomadaire/Section d'hygie 'ne du Secre`tariat de la Socie`te' des Nations $=$ Weekly epidemiological record/Health Section of the Secretariat of the League of Nations. 2004 ;79(4):27.

29. Ravanfar P, Satyaprakash A, Creed R, Mendoza N. Existing antiviral vaccines. Dermatol Ther. 2009;22(2):110-28. doi: 10.1111/j.15298019.2009.01224.x. [PubMed: 19335723].

30. Wellington K, Goa KL. Measles, mumps, rubella vaccine (Priorix; GSKMMR): a review of its use in the prevention of measles, mumps and rubella. Drugs. 2003;63(19):2107-26. doi: 10.2165/00003495 200363190-00012. [PubMed: 12962524].

31. Bovier PA. Recent advances with a virosomal hepatitis A vaccine. Expert Opin Biol Ther. 2008;8(8):1177-85. doi: 10.1517/14712598.8.8.1177. [PubMed: 18613769].

32. Mohebali M, Hajjaran H, Hamzavi Y, Mobedi I, Arshi S, Zarei Z, et al. Epidemiological aspects of canine visceral leishmaniosis in the Islamic Republic of Iran. Vet Parasitol. 2005;129(3-4):243-51. doi: 10.1016/j.vetpar.2005.01.010. [PubMed: 15845279].

33. Sharifi I, FeKri AR, Aflatonian MR, Khamesipour A, Nadim A, Mousavi $\mathrm{MR}$, et al. Randomised vaccine trial of single dose of killed Leishmania major plus BCG against anthroponotic cutaneous leishmaniasis in Bam, Iran. Lancet. 1998;351(9115):1540-3. doi: 10.1016/S01406736(98)09552-X. [PubMed: 10326536].

34. Plotkin SA. Vaccines: the fourth century. Clin Vaccine Immunol 2009;16(12):1709-19. doi: 10.1128/CVI.00290-09. [PubMed: 19793898].

35. Dudek NL, Perlmutter P, Aguilar MI, Croft NP, Purcell AW. Epitope discovery and their use in peptide based vaccines. Curr Pharm Des. 2010;16(28):3149-57. doi: 10.2174/138161210793292447. [PubMed: 20687873].

36. Reed SG, Bertholet S, Coler RN, Friede M. New horizons in adjuvants for vaccine development. Trends Immunol. 2009;30(1):23-32. doi 10.1016/j.it.2008.09.006. [PubMed: 19059004].

37. O'Hagan DT. Recent developments in vaccine delivery systems. Curr Drug Targets Infect Disord. 2001;1(3):273-86. doi 10.2174/1568005014606008. [PubMed: 12455401].

38. Baker PJ. T cell regulation of the antibody response to bacterial polysaccharide antigens: an examination of some general characteristics and their implications. J Infect Dis. 1992;165 Suppl 1:S44-8. doi: 10.1093/infdis/165-Supplement_1-S44. [PubMed:1588176].

39. Gold R, Lepow ML, Goldschneider I, Draper TL, Gotschlich EC. Clinical evaluation of group A and group $\mathrm{C}$ meningococcal polysaccharide vaccines in infants. J Clin Invest. 1975;56(6):1536-47. doi: 10.1172/JCl108235. [PubMed: 1202084].

40. Kayhty H, Karanko V, Peltola H, Makela PH. Serum antibodies after vaccination with Haemophilus influenzae type b capsular polysaccharide and responses to reimmunization: no evidence of immuno- 
logic tolerance or memory. Pediatrics. 1984;74(5):857-65. [PubMed: 6387614].

41. Konradsen HB. Quantity and avidity of pneumococcal antibodies before and up to five years after pneumococcal vaccination of elderly persons. Clin Infect Dis. 1995;21(3):616-20. doi: 10.1093/clinids/21.3.616. [PubMed: 8527553].

42. Avery OT, Goebel WF. Chemo-Immunological Studies on Conjugated Carbohydrate-Proteins : Ii. Immunological Specificity of Synthetic Sugar-Protein Antigens. J Exp Med. 1929;50(4):533-50. doi: 10.1084/jem.50.4.533. [PubMed:19869645].

43. Ward J, Berkowitz C, Pescetti J, Burkart K, Samuelson O, Gordon L. Enhanced immunogenicity in young infants of a new Haemophilus influenzae type $\mathrm{b}$ (Hib) capsular polysaccharide (PRP)-diphtheria toxoid (D) conjugate vaccine. Pediatr Res. 1984;18(287).

44. Peltola H, Kilpi T, Anttila M. Rapid disappearance of Haemophilus influenzae type $\mathrm{b}$ meningitis after routine childhood immunisation with conjugate vaccines. Lancet. 1992;340(8819):592-4. doi: 10.1016/0140-6736(92)92117-X. [PubMed:1355165].

45. Plotkin SA. Vaccines: past, present and future. Nat Med. 2005;11(4 Suppl):S5-11. doi: 10.1038/nm1209. [PubMed:15812490].

46. Nascimento IP, Leite LC. Recombinant vaccines and the development of new vaccine strategies. Braz J Med Biol Res. 2012;45(12):1102-11. doi: 10.1590/S0100-879X2012007500142. [PubMed: 22948379].

47. Abbas AK, Lichtman AH, Pillai S. Cellular and molecular immunology. Elsevier Health Sciences; 2014.

48. Palatnik-de-Sousa CB. Vaccines for leishmaniasis in the fore coming 25 years. Vaccine. 2008;26(14):1709-24. doi: 10.1016/j.vaccine.2008.01.023. [PubMed: 18295939]
49. Borja-Cabrera GP, Santos FB, Nico D, Gravino AE, Manna L, Palatnik M, et al. The Leishmune ${ }^{\circ}$ `s Nucleoside hydrolase DNA vaccine as an aid in immunotherapy of canine visceral leishmaniasis. Procedia Vaccinol. 2012;6:64-73. doi:10.1016/j.provac.2012.04.009.

50. Smillie C, Garcillan-Barcia MP, Francia MV, Rocha EP, de la Cruz F. Mobility of plasmids. Microbiol Mol Biol Rev. 2010;74(3):434-52. doi: 10.1128/MMBR.00020-10. [PubMed: 20805406].

51. Fındık A. , Çiftci A. . Bacterial DNA vaccines in veterinary medicine: A review. J Vet Adv. 2012;2(4):139-48.

52. Bins AD, van den Berg JH, Oosterhuis K, Haanen JB. Recent advances towards the clinical application of DNA vaccines. Neth J Med. 2013;71(3):109-17. [PubMed: 23712805].

53. Tahamtan A, Ghaemi A, Gorji A, Kalhor HR, Sajadian A, Tabarraei A, et al. Antitumor effect of therapeutic HPV DNA vaccines with chitosan-based nanodelivery systems. J Biomed Sci. 2014;21:69. doi: 10.1186/s12929-014-0069-z. [PubMed: 25077570].

54. Tahamtan A, Tabarraei A, Moradi A, Dinarvand M, Kelishadi M, Ghaemi A, et al. Chitosan nanoparticles as a potential nonviral gene delivery for HPV-16 E7 into mammalian cells. Artif Cells Nanomed Biotechnol. 2015;43(6):366-72. doi: 10.3109/21691401.2014.893522. [PubMed: 24641772].

55. Rappuoli R, Medaglini D. Big science for vaccine development. Vaccine. 2014;32(37):4705-7. doi: 10.1016/j.vaccine.2014.06.071. [PubMed: 24975815].

56. Tahamtan A, Char Ostad J, Behboudi E, Hoseini-Shokouh SJ, Barati M. Nanotechnology and Modern Medicine: The medical applications of nanotechnology [In Persian]. Nurse Phys Within War. 2016;4(10):80-90. 